\title{
Simulation of Control Structures for Slug Flow in Riser during Oil Production
}

\author{
Ole Magnus Brastein Roshan Sharma \\ Department of Electrical Engineering, IT, and Cybernetics, University College of Southeast Norway, Porsgrunn, \\ Norway, \{ole.m.brastein, roshan.sharma\}@usn.no
}

\begin{abstract}
The occurrence of slug flow is a common problem arising in the oil well riser pipeline. To eliminate such slug flow, various control structures along with state estimation are designed and compared in this paper. Nonlinear model based predictive scheme are compared with classical PI controllers for three different control structures. One of the control structure is based on controlling the mass of the liquid in the riser pipeline, for which, an Unscented Kalman Filter is designed to estimate the mass. The simulation results show that the model based controllers perform relatively better than the classical controllers. Although computationally expensive, the control algorithm used in this paper for model based control still makes it real time implementable.
\end{abstract}

Keywords: slug flow, oil riser, model based control, PI control, unscented Kalman filter

\section{Introduction}

In oil well riser pipelines with low-point angle, the liquid column accumulated in the riser above the low point acts as a virtual valve (see Figure 1), alternately blocking and letting the gas produced from the reservoir to flow through the riser. This is due to the hydrostatic pressure exerted at the low point by the liquid column in the riser. The gas produced from the reservoir at first starts to accumulate below the low point. The pressure builds up and reaches to a critical point where the built-up pressure exceeds the hydrostatic pressure drop. This results in a rapid discharge of the accumulated gas to the riser. This large gas bubble/volume pushes up some of the liquid in the riser and out from the choke valve. However, with time, the liquid again starts to accumulate in the riser. The gas pressure at the horizontal flowline starts to build up again and the cycle repeats. This behavior of the fluid flow in the riser is known as slug flow. It is an unstable multiphase flow where oscillation in the production of oil from the reservoir occurs.

Formation of slug flow in oil well riser pipelines has been studied by many other researchers. Various control solutions to avoid the slug flow in oil wells along with the development of mathematical model and controllability analysis can be found at (Storkaas, 2005). Control of riser base pressure for stabilizing the slug flow have been studied by (Aamo et al., 2005; Dalsmo et al., 2005). A simplified model based on first principles modeling for reproducing slugging oscillations of a real oil well was developed by (Meglio et al., 2009). This model was later used by (Meglio et al., 2012b) for designing control structures. Control strategies for slug control and tuning rules was studied by (Godhavn et al., 2005). A nonlinear controller using integrator backstepping approach was used by (Kaasa et al., 2007) to stabilize unstable wells. A review of recent advances in the suppression of the slugging phenomenon by model-based control can be found at (Meglio et al., 2012a). This article gives a clear presentation of the evaluation and comparison of the existing solutions and proposes directions for improvement. A similar type of slugging phenomenon is also observed for gaslifted oil wells. Stabilization of gas-lift wells by feedback control was studied by (Eikrem, 2006; Imsland, 2002). An insight and understanding into how feedback control can be used to avoid severe slugging, thereby bridging the gap between control and petroleum engineering can be found at (Havre and Dalsmo, 2002).

In this paper, three different control structures/strategies are developed to stabilize the flow in the riser so that the flow does not oscillate and becomes stable. In Section 2, a brief description of the model that captures the slug flow phenomenon in the riser is described. The model is simulated to illustrate its capability of capturing the formation of slug flow in the riser in Section 3. Implementation of an Unscented Kalman Filter(UKF) for estimating the states of the system is provided in Section 4. In Section 5, the formulation of the three different control strategies is presented. The simulation results obtained from the model based controller and the PI controller are clarified, compared and discussed in Section 6. A possibility for the real time implementation and the computational time required by the model predictive controller (MPC) is discussed in Section 7. A brief discussion on the maximum valve opening that can be achieved before the flow becomes unstable again is presented in Section 8. Finally, conclusions are provided in Section 9.

\section{Model for slug flow}

A widely used mathematical model for representing the slug flow in the oil well risers was developed by (Meglio et al., 2012b) and this model has been used in the present 


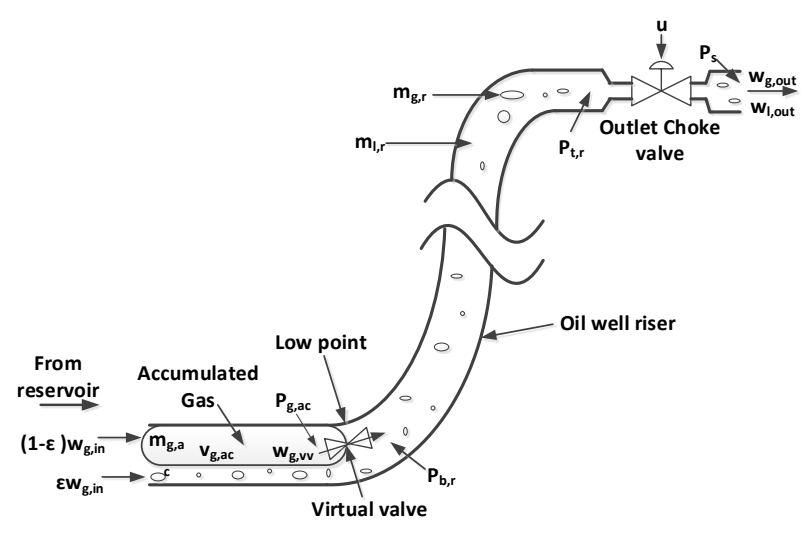

Figure 1. Schematic of fluid transportation in the flow line and riser.

work. Only a brief description of the model is presented in this section and the details about the development of the model can be found at (Meglio et al., 2012b). Let us consider the low point of the riser at the place where the virtual valve is located as shown in Figure 1.

The flowline and the riser are divided into three separate volumes/parts: (i) Volume at the horizontal part of the flowline where the incoming gas from the reservoir accumulates, (ii) Volume of the vertical riser filled with liquid only, and (iii) Volume of the vertical riser filled with gas only. The model is based on the conservation principle where the mass balance is applied to each of the three volumes. The state variables are the mass of the gas accumulated in the horizontal flowline $\left(m_{g, a c}\right)$, the mass of the gas in the riser $\left(m_{g, r}\right)$ and the mass of the liquid in the riser $\left(m_{g, l}\right)$. From the mass balances we obtain,

$$
\begin{gathered}
\frac{d m_{g, a c}}{d t}=(1-\lambda) w_{g, \text { in }}-w_{g, v v}, \\
\frac{d m_{g, r}}{d t}=\lambda w_{g, \text { in }}+w_{g, v v}-w_{g, \text { out }}, \\
\frac{d m_{g, l}}{d t}=w_{l, \text { in }}-w_{l, \text { out }} .
\end{gathered}
$$

Here, $\lambda$ denotes the fraction of gas coming from the reservoir that directly flows to the riser, $w_{g, \text { in }}$ is the flow rate of the gas entering the riser, $w_{g, v v}$ is the flow rate of the gas through the virtual valve, $w_{g, \text { out }}$ is the flow rate of the gas flowing out of the riser through the outlet choke valve, $w_{l, \text { in }}$ is the flow rate of the liquid entering the riser and $w_{l, \text { out }}$ is the flow rate of the liquid flowing out of the riser through the outlet valve. The algebraic equations involved in the models are listed below. These are taken from (Meglio et al., 2012b) and the details about their development is not provided in this paper.

$$
\begin{gathered}
w_{g, v v}=C_{g, v v} \max \left(0,\left(P_{g, a c}-P_{b, r}\right)\right) \\
P_{g, a c}=\frac{m_{g, a c} R T}{M V_{g, a c}}
\end{gathered}
$$

$$
\begin{gathered}
V_{g, r}=V_{r}-\frac{\left(m_{l, r}+m_{l, \text { min }}\right)}{\rho_{l}} \\
P_{t, r}=\frac{m_{g, r} R T}{M V_{g, r}} \\
P_{b, r}=P_{t, r}+\left(m_{l, r}+m_{l, \text { min }}\right) \frac{g \sin \theta}{A} \\
w_{\text {out }}=C_{\text {out }} u \sqrt{\rho_{m}\left(P_{t, r}-P_{s}\right)} \\
w_{l, \text { out }}=\frac{m_{l, r}}{m_{l, r}+m_{g, r}} w_{\text {out }} \approx w_{\text {out }} \\
w_{g, \text { out }}=\frac{m_{g, r}}{m_{l, r}+m_{g, r}} w_{\text {out }} \approx \frac{m_{g, r}}{m_{l, r}} w_{\text {out }}
\end{gathered}
$$

Here, $C_{g, v v}$ is the valve constant for the virtual valve, $P_{g, a c}$ is pressure of the gas accumulated in the horizontal flowline (upstream the virtual valve), $P_{b, r}$ is the pressure at the bottom of the riser, $P_{t, r}$ is the pressure at top of the riser, $P_{S}$ is the separator pressure, $R$ is the ideal gas constant, $T$ is the temperature of the fluid in the riser, $M$ is the molar mass of the gas, $V_{g, a c}$ is the volume of the horizontal flowline where the gas accumulates, $V_{r}$ is the physical volume of the riser, $V_{g, r}$ is the volume of gas in the riser, $m_{l, \min }$ is the minimum amount of liquid present in the riser at all times, $\rho_{l}$ is the density of the liquid, $\theta$ is the mean inclination of the riser pipe, $A$ is the cross section of the riser, $C_{\text {out }}$ is the valve constant for the outlet choke valve, $u \in[0,1]$ is the valve opening and $w_{\text {out }}$ is the total mass flow rate flowing out of the riser through the outlet valve.

\section{Simulation for slug flow}

The model presented in Section 2 is simulated in MATLAB to observe the occurrence of the slug flow in the riser pipeline. The mass flow rate of the gas and the liquid flowing into the well from the reservoir are considered to be constant. With a nominal valve opening of 0.35 or $35 \%$, the fluid flow in the riser pipe i.e. the outflow from the outlet valve exhibits a slug flow as shown in Figure 2.

The flow of the liquid from the outlet valve oscillates with a time period of about 50 minutes. This is due to the virtual valve that alternately blocks and lets the gas to flow through the riser, thus producing a slug flow. The pressure at the bottom and top of the riser oscillates and this oscillating nature of the pressures in the riser actually creates the slug flow. The average production of oil from the field due to an unstable slug flow is lower than the theoretical steady state (or equilibrium) production. Such unstable slug flow should be controlled or stabilized.

In reality, the process operators choke the outlet valve manually to stabilize the slug flow. The slug flow can be stabilized by decreasing the opening of the output choke valve. A stabilized slug flow results in a non-oscillating pressure at the bottom of the riser. In Figure 3, the choke valve opening is decreased from $45 \%$ to $15 \%$ in steps. As can be seen from Figure 3, the pressure at the bottom of the riser still keeps on oscillating when the valve opening 

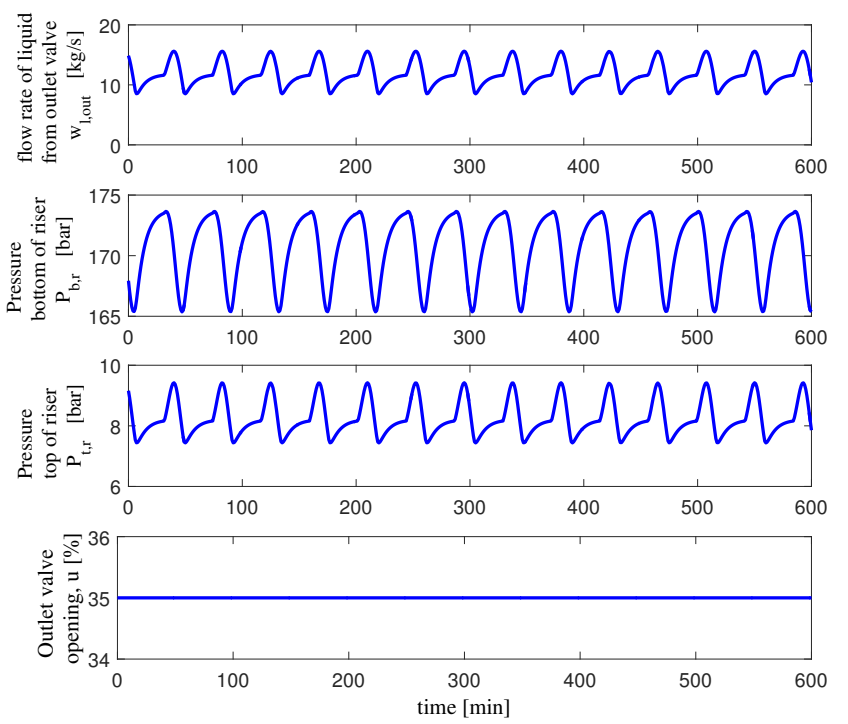

Figure 2. Slug flow in the riser for $\mathrm{u}=35 \%$.

is reduced from $45 \%$ to $25 \%$. At time $600 \mathrm{~min}$, the outlet valve opening is reduced to $15 \%$. With this valve opening, the pressure at the bottom of the riser is stabilized and this results in a stabilized flow of the liquid through the outlet valve. As the valve is slowly choked, at one point, the flow is stabilized. This value of valve opening for which the flow starts to stabilize is called the bifurcation point. Above this point, the flow is unstable and below this point, the flow is stable. From the openloop simulations, the bifurcation point was found out to be around $20 \%$ valve opening.

From Figure 3, we can observe that the flow can be stabilized by remaining below the bifurcation point in the stable region. Usually, the bifurcation point corresponds to a low valve opening. With a lower valve opening, the flow rate of the oil produced from the well is also low which is economically not beneficial. Thus, it is of interest to investigate whether the flow can be stabilized by opening the valve in the unstable zone (i.e. by remaining above the bifurcation point) through the use of different control strategies.

\section{State estimation}

One of the control structure that is explained in detail in Section 5 utilizes the information about the mass of the liquid (which is a state variable) in the riser pipeline. This and the remaining two states of the process cannot be directly measured and hence should be estimated. For this, an Unscented Kalman Filter (UKF) that directly utilizes the nonlinear model of the process is implemented. Details of the UKF is not the main focus of this paper and interested readers are advised to follow (Simon, 2006). In this work, standard algorithm for UKF available at (Simon, 2006) is implemented in MATLAB.

In addition, it is assumed that the pressures at the bot-
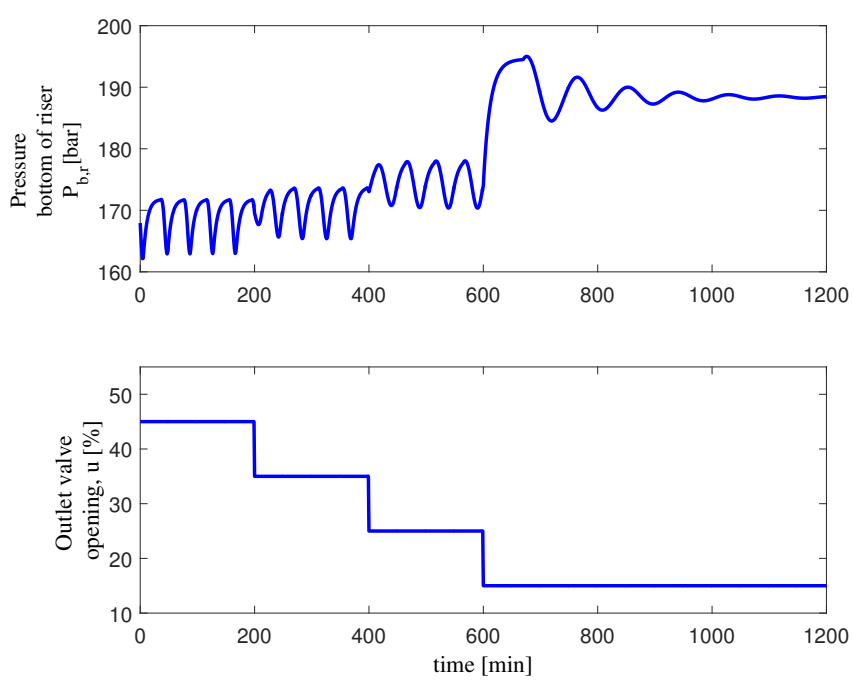

Figure 3. Variation of pressure at the bottom of the riser with valve opening.

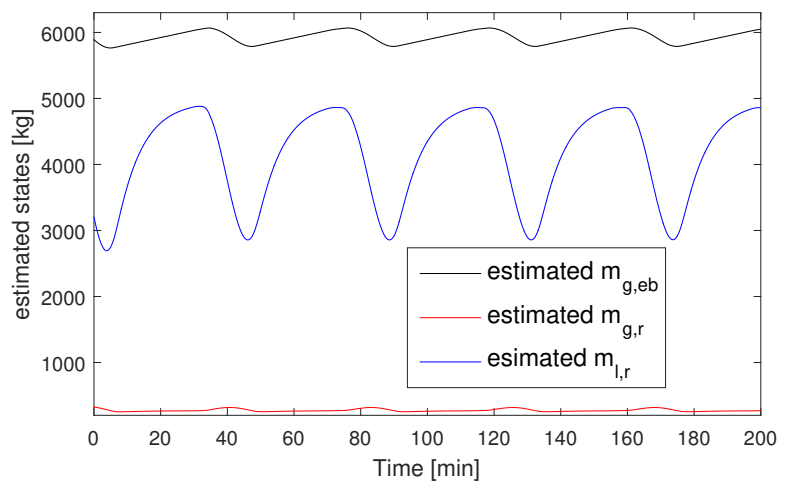

Figure 4. Estimated states by UKF.

tom and top of the riser pipe are measured and available. Figure 4 shows the estimated states of the system and Figure 5 shows the estimated pressures at the bottom and top of the riser pipeline. The estimated states and the estimated measurements are then used by the control structures for stabilizing the flow.

\section{Control strategies}

For regulating the slug flow in the riser pipeline, three different control strategies/structures were developed.

- The first control structure stabilizes the slug flow by controlling the pressure at the bottom of the riser i.e. by controlling $P_{b, r}$ to a set point.

- The second control structure stabilizes the slug flow by controlling the pressure drop in the riser i.e. by controlling $\triangle P=\left(P_{b, r}-P_{t, r}\right)$ to a set point.

- The third control structure stabilizes the slug flow by controlling the total mass of the liquid in the riser i.e. by controlling $m_{l, r}$ to a setpoint. 

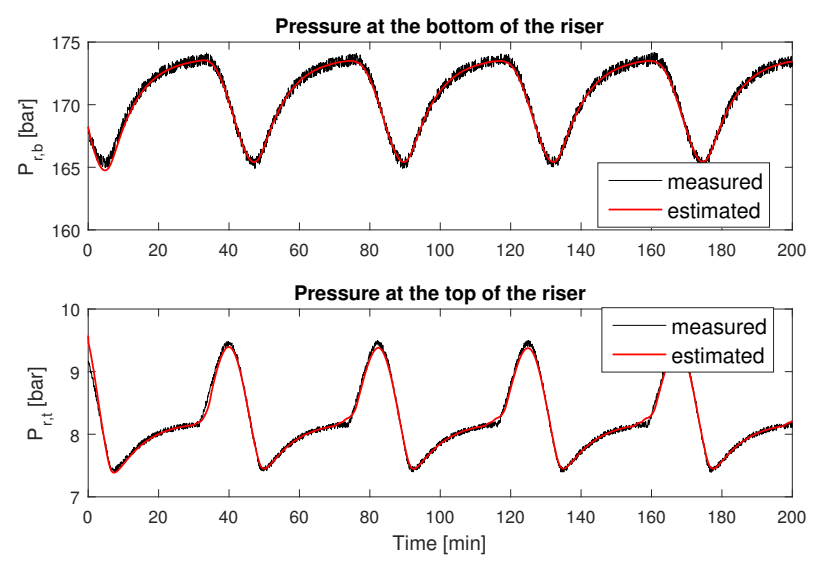

Figure 5. Estimated pressures at the bottom and top of the riser.

Each of these control strategies were implemented as a model based controller using model predictive control (MPC) scheme as well as using a standard ProportionalIntegral (PI) control scheme.

\subsection{Model predictive control}

For designing a nonlinear model predictive controller, consider the following nonlinear objective function,

$$
\begin{array}{r}
\min _{\triangle u_{c}} f\left(\triangle u_{c}\right)=\sum_{k=1}^{N_{p}}\left(X X_{k}-X X_{k}^{r e f}\right)^{T} P_{k}\left(X X_{k}-X X_{k}^{r e f}\right) \\
+\sum_{k=1}^{N_{c}}\left(\triangle u_{k}\right)^{T} R_{k}\left(\triangle u_{k}\right)
\end{array}
$$

Here, $X X_{k}$ is the variable to be controlled and $X X_{k}^{r e f}$ is its reference value depending on the choice of the control structure. $X X_{k}=P_{b, r}$ for control structure $1, X X_{k}=$ $\triangle P=\left(P_{b, r}-P_{t, r}\right)$ for control structure 2 and $X X_{k}=m_{l, r}$ for control structure 3. $N_{p}$ is the prediction horizon length and $N_{c}$ is the control horizon length. $P_{k}$ is the weighting factor for the set point error and $R_{k}$ is the weighting factor for the control deviation. $\triangle u_{k}=u_{k}-u_{k-1}$ is the rate of change of control action.

The choke valve opening should be between 0 and 1 , i.e. the constraint in the control input is,

$$
0 \leq u_{k} \leq 1
$$

In practice, the choke valves are opened in smaller steps, and larger abrupt changes in its opening is usually avoided. Consider that the choke valve can be opened or closed by only $0.5 \%$ per second i.e.

$$
-0.5 \% \leq \triangle u_{k} \leq 0.5 \%
$$

(13) and (14) together with the model of the process form the constraints for the optimization problem. Moreover, in order to improve the speed of computation without loosing any control dynamics, the prediction horizon was grouped into four groups.
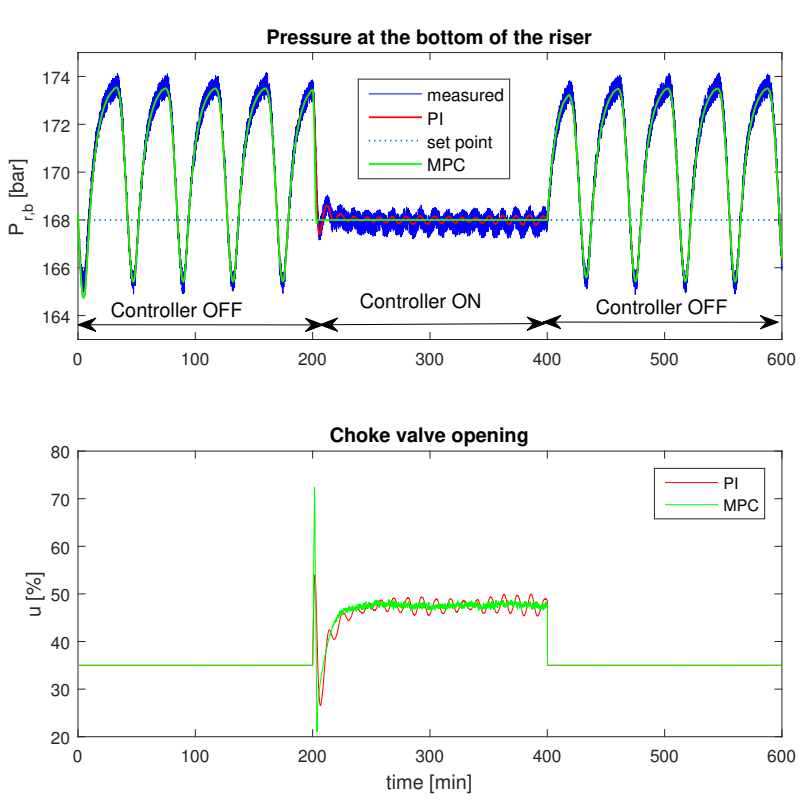

Figure 6. Pressure at the bottom of the riser with control structure 1 .

\subsection{PI control}

A standard expression for the PI controller in the deviation form can be written as,

$$
\triangle u_{k}=K_{p}\left(1+\frac{d t}{2 T_{i}}\right) e_{k}-K_{p}\left(1-\frac{d t}{2 T_{i}}\right) e_{k-1}
$$

with

$$
e_{k}=X X_{k}^{r e f}-X X_{k},
$$

corresponding to the three control structures respectively. The conditions fulfilling (13) and (14) were implemented together with (15) and (16). Here, $K_{p}$ is the proportional gain of the controller, $T_{i}$ is the integral time constant and $d t$ is the sampling time taken to be 5 seconds.

\section{Simulation results and discussion}

\subsection{Control structure I}

The set point for the pressure at the bottom of the riser was chosen to be 168 bar. Figure 6 and Figure 7 show the simulation results both with the model predictive controller and with the PI controller. Each controller was turned on between the time interval of 200 and 400 minutes. Both controllers were able to stabilize the outflow (see Figure 7) however, the performance of the model predictive controller was better than the PI controller. Small oscillations of the pressure at the bottom of the riser and in the choke valve opening were seen with the PI controller. However with MPC, such small oscillations were completely eliminated.

From the openloop simulations it is known that the bifurcation point of the valve opening is around $20 \%$. However, with this control structure, the valve remains opened 


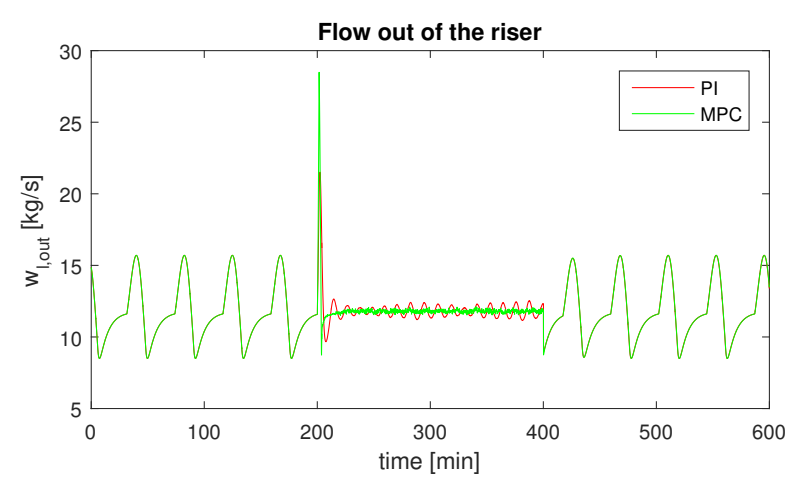

Figure 7. Stabilization of outflow with control structure 1.
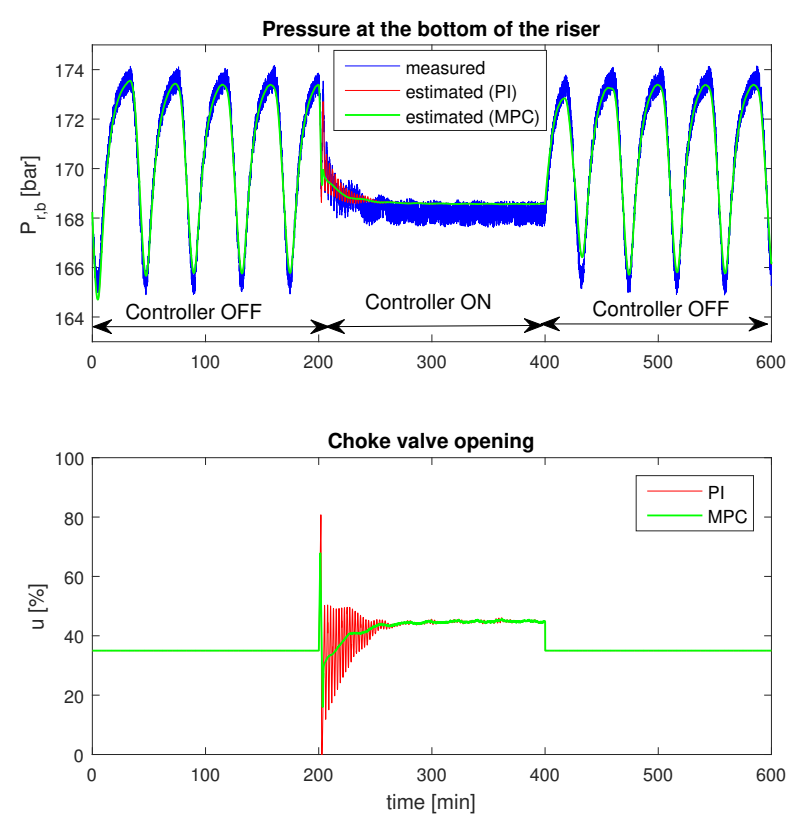

Figure 8. Pressure at the bottom of the riser with control structure 2.

at around $48 \%$ which is in the unstable region of the valve opening. This clearly indicates that it is possible to stabilize the flow flowing in the riser pipeline while still staying at the unstable region of the valve opening. This is an added benefit with respect to the operation of the process: the more the valve opening, the more is the amount of oil flowing out of the well (economically more beneficial).

\subsection{Control structure II}

For control structure II, the set point for the pressure difference between the bottom and the top of the riser was taken to be 161 bar. Figure 8 and Figure 9 show the simulation results both with the model based controller and with the PI controller. Both control schemes were able to stabilize the slug flow. The simulation results show that the bottom hole pressure can be indirectly stabilized by controlling the pressure drop over the riser. However, the model based controller outperforms the PI controller. High frequency oscillations in the valve opening

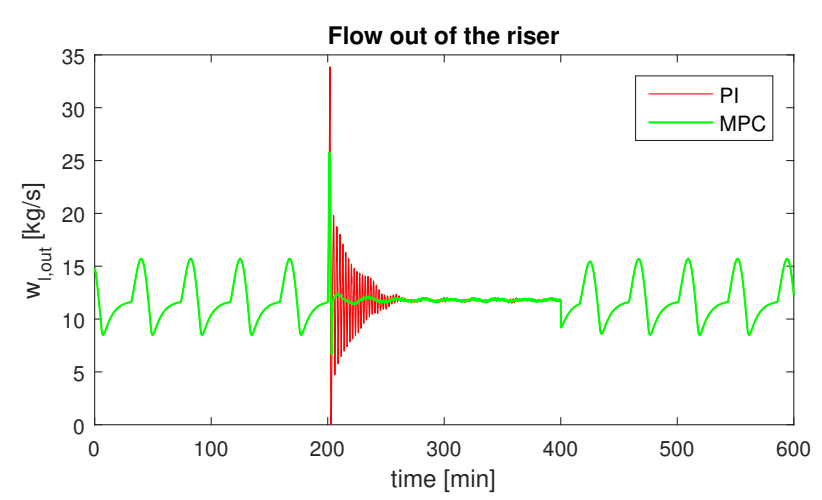

Figure 9. Stabilization of outflow with control structure 2.

(and in the flow through the choke valve) were observed at the beginning of the control action with the PI controller, while such oscillations were completely suppressed by the model predictive controller. The control scheme utilizes the estimated values calculated by the UKF. If the controller uses the measurement of the pressures directly for the calculation of control actions, the dynamics become even more oscillatory (with higher frequency oscillations). Therefore for this control structure, proper tuning of the UKF is very essential. Compared to control structure 1, more weight (10 times more) was put to the measurement noise covariance matrix during the implementation of UKF.

\subsection{Control structure III}

With this control structure, the mass of the liquid in the riser was controlled to a set point of $3800 \mathrm{~kg}$. Since the mass of the liquid in the riser has a significant and a direct effect on the pressure at the bottom of the riser, controlling it allows the bottom hole pressure to be controlled indirectly. Figure 10 and Figure 11 show the simulation results. It is very clear that for this control structure, the PI controller does not stabilize the flow properly. The model predictive controller outperforms the PI controller. Although the amplitude of the oscillation for the pressure at the bottom of the riser was lowered with the PI controller (which is better than without any control at all), but at the same time the frequency of the oscillation was increased. The valve openings oscillated periodically and the flow of the fluid through it oscillated with a more higher frequency than before (without any control). However, with the model predictive controller, the control action was superior without any oscillations. The flow of fluid out of the riser and the pressure at the bottom of the riser were very stable.

\section{Computational time for MPC}

Model predictive controller is a computationally heavy algorithm. At each iteration, a constrained nonlinear optimization problem is solved. In this work, a prediction horizon of 30 samples with a sampling time of $5 \mathrm{sec}$ which equals to $150 \mathrm{sec}$ was used. The control inputs 

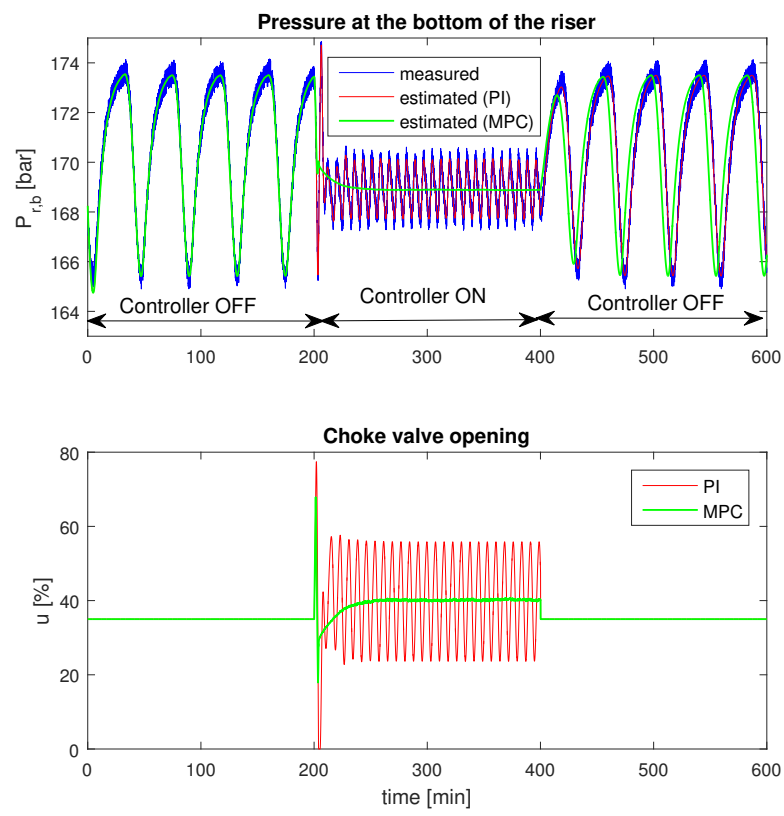

Figure 10. Pressure at the bottom of the riser with control structure 3 .

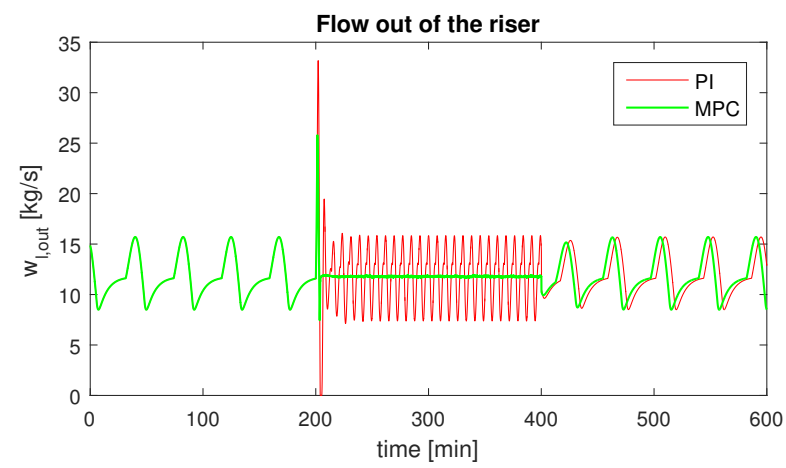

Figure 11. Stabilization of outflow with control structure 3.

were grouped into four groups. Thus, instead of optimizing 30 unknowns at each iteration, only 4 unknowns are optimized. This significantly reduces the computational time without deteriorating the control action. For all the three control structures, each iteration could be solved in less than a second, and for the chosen sampling interval, this means that the algorithm can be easily implemented for real time application. For an illustration, the computational time required by the MPC algorithm for control structure 2 is shown in Figure 12. A normal computer with $2.50 \mathrm{Ghz}$ processor and $4 \mathrm{~GB}$ RAM was used for the simulations.

\section{Maximum valve opening}

The choice of the setpoint(s) for all the three control structures were arbitrary and can be freely chosen. However, the choice of the setpoint influences the ability of the controller to stabilize the flow. To illustrate this, consider the

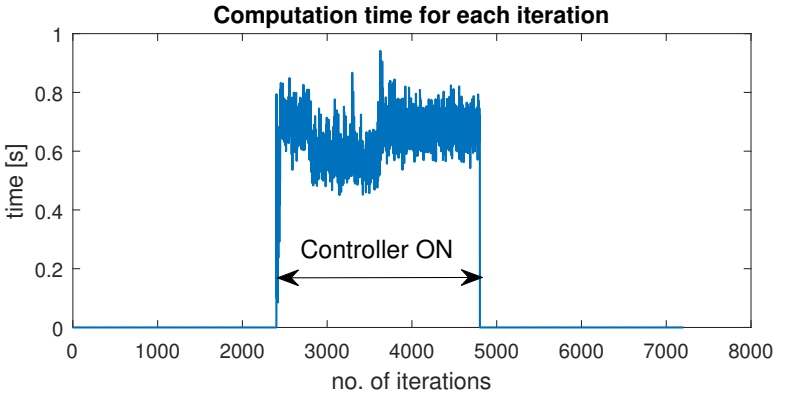

Figure 12. Computational time required by the model predictive controller for structure 2.
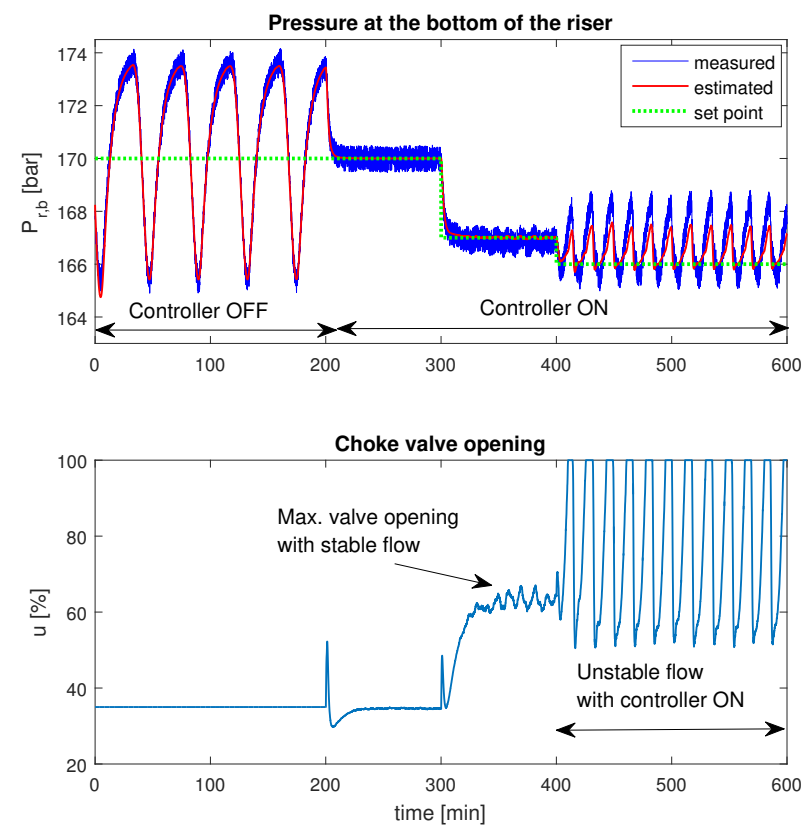

Figure 13. Maximum valve opening for stable flow with control structure 1.

first control structure. Lowering the value of the setpoint for the pressure at the bottom of the well corresponds to a higher valve opening and hence more flow of oil through the outlet choke valve as shown in Figure 13. With respect to the production of oil, it is economically beneficial to have the outlet valve open as much as possible. In Figure 13, as the setpoint was decreased from 170 bar to 167 bar, the valve opening was increased from $35 \%$ to $65 \%$ (maximum opening that marks the boundary between the stable and the unstable flow). As the setpoint was further decreased to $166 \mathrm{bar}$ after $400 \mathrm{~min}$, the pressure started to oscillate and the flow became unstable again, even though the controller was still turned on.

The presence of a maximum valve opening before the flow again becomes unstable was also seen with the other two control structures but have not been shown in this paper to save space. 


\section{Conclusions}

From this research work, it was observed that the model predictive control schemes outperform the standard PI controllers for stabilizing the slug flow in oil well riser. The differences are not so significant for the first two control strategies. However, for the third control strategy, the control actions are superior with the model based control. Nonlinear MPC with Unscented Kalman filter can be implemented for real time control of the slug flow in riser. Among the three control structures, with the model predictive control, all the control structures were equally able to stabilize the flow. It is difficult to conclude which of these control structure is the best with the model predictive control. However, with the PI controllers, the first control strategy stabilized the flow better than the remaining two control structures.

\section{References}

O. M. Aamo, G. O. Eikrem, H. B. Siahaan, and B. A. Foss. Observer design for multiphase flow in vertical pipes with gas-lift theory and experiments. Journal of Process Control, 15:247-257, 2005.

M. Dalsmo, E. Halvorsen, and O. Slupphaug. Active feedback control of unstable wells at the brage field. Modeling, Identification and Control, 26(2):81-94, 2005. doi:10.4173/mic.2005.2.2.

G. O. Eikrem. Stabilization of gas-lift wells by feedback control. $\mathrm{PhD}$ thesis, Norwegian University of Science and Technology, 2006.

J. M. Godhavn, M. P. Fard, , and P. H. Fuchs. New slug control strategies, tuning rules and experimental results. Journal of Process Control, 15:547-557, 2005.

K. Havre and M. Dalsmo. Active feedback control as a solution to severe slugging. volume 17, New Orleans, Lousiana, 2002. Society of Petroleum Engineers. doi:10.2118/79252-PA.

L. S. Imsland. Output Feedback and Stabilization and Control of Positive Systems. PhD thesis, Norwegian University of Science and Technology, Department of Engineering Cybernetics, Trondheim, Norway, 2002.

Glenn-Ole Kaasa, Vidar Alstad, Jing Zhou, and Ole Morten Aamo. Nonlinear model-based control of unstable wells. Modeling, Identification and Control, 28(3):69-79, 2007.

F. D. Meglio, G. O. Kaasa, N. Petit, and V. Alstad. Reproducing slugging oscillations of a real oil well. In $49^{\text {th }}$ IEEE Conference on Decision and Control, pages 4473-4479, Hilton Atlanta Hotel, Atlanta, GA, USA, December 15-17 2009.

F. D. Meglio, G. O. Kaasa, N. Petit, and V. Alstad. Modelbasedcontrolofslugging:advancesand challenges. In 2012 IFAC Workshop on Automatic Control in Offshore Oil and Gas Production, pages 109-115, Norwegian University of Science and Technology, Trondheim, Norway, May 31- June $12012 \mathrm{a}$.
F. D. Meglio, N. Petit, V. Alstad, and G. O. Kaasa. Stabilization of slugging in oil production facilities with or without upstream pressure sensors. Journal of Process Control, 22: 809-822, 2012b. doi:10.1016/j.jprocont.2012.02.014.

D. Simon. Optimal State Estmation: Kalman, $H_{\infty}$ and Nonlinear Approaches. John Wiley \& Sons, Inc., 2006.

E. Storkaas. Control Solutions to Avoid Slug Flow in Pipelineriser Systems. PhD thesis, Norwegian University of Science and Technology, 2005. 